\title{
DECIDABILITY AND COMPLEXITY IN AUTOMATIC MONOIDS*
}

\author{
MARKUS LOHREY \\ FMI, University of Stuttgart, Germany \\ lohrey@i1.informatik.rwth-aachen.de \\ Received (received date) \\ Revised (revised date) \\ Communicated by Editor's name
}

\begin{abstract}
Several complexity and decidability results for automatic monoids are shown: (i) there exists an automatic monoid with a P-complete word problem, (ii) there exists an automatic monoid such that the first-order theory of the corresponding Cayley-graph is not elementary decidable, and (iii) there exists an automatic monoid such that reachability in the corresponding Cayley-graph is undecidable. Moreover, it is shown that for every hyperbolic group the word problem belongs to LOGCFL, which improves a result of Cai [8].

Keywords: automatic monoids, hyperbolic groups, word problems, Cayley-graphs, complexity, decidability
\end{abstract}

\section{Introduction}

Automatic groups attracted a lot of attention in combinatorial group theory during the last 15 years, see e.g. the textbook [15]. Roughly speaking, a finitely generated group $\mathcal{G}$, generated by the finite set $\Gamma$, is automatic, if the elements of $\mathcal{G}$ can be represented by words from a regular language over $\Gamma$, and the multiplication with a generator can be recognized by a synchronized 2-tape automaton. This concept easily yields a quadratic time algorithm for the word problem of an automatic group.

It is straight forward to extend the definition of an automatic group to the monoid case; this leads to the class of automatic monoids, see e.g. [10, 18, 22, 36]. In the present paper, we study the complexity and decidability of basic algorithmic questions in automatic monoids. In Section 4 we consider the complexity of the word problem for automatic monoids. Analogously to the group case, it is easy to show that for every automatic monoid the word problem can be solved in quadratic time. Here, we prove that there exists a fixed automatic monoid with a P-complete word problem. Thus, unless $\mathrm{P}=\mathrm{NC}$, where $\mathrm{NC}$ is the class of all problems that can be solved in polylogarithmic time using a polynomial amount of hardware, there exist

\footnotetext{
${ }^{*}$ This work was partly done while the author was at RWTH Aachen, Germany.
} 
automatic monoids for which the word problem cannot be efficiently parallelized. Whether there exists an automatic group with a P-complete word problem was asked for the first time by Cai [8]. This problem remains open.

An important subclass of the class of automatic groups is the class of hyperbolic groups, which are defined via a geometric hyperbolicity condition on the Cayleygraph [16]. In [8], Cai has shown that for every hyperbolic group the word problem belongs to the parallel complexity class $\mathrm{NC}^{2}$. Cai also asked, whether the upper bound of $\mathrm{NC}^{2}$ can be improved. Using known results from formal language theory, we show in Section 4 that the word problem for every hyperbolic group belongs to the complexity class LOGCFL $\subseteq \mathrm{NC}^{2}$. LOGCFL is the class of all problems that are logspace reducible to a context-free language [44]. We also present a class of automatic monoids, namely monoids that can be presented by finite, terminating, confluent, and left-basic semi-Thue systems [41], for which the complexity of the word problem captures the class LOGDCFL (the logspace closure of the deterministic context-free languages [44]).

In Section 5 we study Cayley-graphs of automatic monoids. The Cayley-graph of a finitely generated monoid $\mathcal{M}$ with respect to a finite generating set $\Gamma$ is a $\Gamma$-labeled directed graph with node set $\mathcal{M}$ and an $a$-labeled edge from a node $x$ to a node $y$ if $y=x a$ in $\mathcal{M}$. Cayley-graphs of groups are a fundamental tool in combinatorial group theory [32] and serve as a link to other fields like topology, graph theory, and automata theory, see, e.g., [34, 35]. Results on the geometric structure of Cayley-graphs of automatic monoids can be found in [42, 43]. Here we consider Cayley-graphs from a logical point of view, see $[26,27]$ for previous results in this direction. More precisely, we consider the first-order theory of the Cayley-graph of an automatic monoid $\mathcal{M}$. This theory contains all true statements of the Cayley-graph that result from atomic statements of the form "there is an $a$ labeled edge between two nodes" using Boolean connectives and quantification over nodes. From the definition of an automatic monoid it follows immediately that the Cayley-graph of an automatic monoid is an automatic graph in the sense of [3, 24]; hence, by a result from [24], its first-order theory is decidable. This allows to verify non-trivial properties for automatic monoids, like for instance right-cancellativity, which are undecidable for arbitrary monoids. Here, we prove that there exists an automatic monoid such that the first-order theory of the corresponding Cayleygraph is not elementary decidable. This result sharpens a corresponding statement for general automatic graphs [3]. We remark that, using a result from [30], the Cayley-graph of a right-cancellative automatic monoid has an elementarily decidable first-order theory. Finally we prove that there exists an automatic monoid $\mathcal{M}$ such that reachability in the Cayley-graph (i.e., the question whether for given monoid elements $u$ and $v$ there exists $x \in \mathcal{M}$ with $u=v x$ in $\mathcal{M}$ ) is undecidable.

The short version of this paper can be found in [31].

\section{Monoids and Word Problems}

More details and references concerning the material in this section can be found in [7]. In the following, let $\Gamma$ be always a finite alphabet of symbols. A semi-Thue 
system $R$ over $\Gamma$ is a (not necessarily finite) set $R \subseteq \Gamma^{*} \times \Gamma^{*}$; its elements are called rules. A rule $(s, t)$ will be also written as $s \rightarrow t$. Without loss of generality we may assume that every symbol from $\Gamma$ appears in a rule of $R$; thus, $\Gamma$ is given uniquely by $R$. Let $\operatorname{dom}(R)=\{\ell \mid \exists r:(\ell, r) \in R\}$ and $\operatorname{ran}(R)=\{r \mid \exists \ell:(\ell, r) \in R\}$. We define the binary relation $\rightarrow_{R}$ on $\Gamma^{*}$ by: $x \rightarrow_{R} y$ if $\exists u, v \in \Gamma^{*} \exists(s, t) \in R: x=u s v$ and $y=u t v$. Let $\stackrel{*}{\leftrightarrow}_{R}$ be the smallest equivalence relation on $\Gamma^{*}$ containing $\rightarrow_{R}$; it is a congruence with respect to the concatenation of words and called the Thuecongruence associated with $R$. Hence, we can define the quotient monoid $\Gamma^{*} / \stackrel{*}{\leftrightarrow}_{R}$, which is briefly denoted by $\Gamma^{*} / R$. Let $\pi_{R}: \Gamma^{*} \rightarrow \Gamma^{*} / R$ be the canonical surjective monoid homomorphism that maps a word $w \in \Gamma^{*}$ to its equivalence class with respect to $\stackrel{*}{\leftrightarrow}_{R}$. A monoid $\mathcal{M}$ is finitely generated if it is isomorphic to a monoid of the form $\Gamma^{*} / R$. In this case, we also say that $\mathcal{M}$ is finitely generated by $\Gamma$. If in addition to $\Gamma$ also $R$ is finite, then $\mathcal{M}$ is a finitely presented monoid. The word problem of $\mathcal{M} \simeq \Gamma^{*} / R$ with respect to $R$ is the set $\left\{(u, v) \in \Gamma^{*} \times \Gamma^{*} \mid \pi_{R}(u)=\pi_{R}(v)\right\}$; it is undecidable in general $[33,37]$. If a monoid $\mathcal{M}$ is isomorphic to both $\Gamma^{*} / R$ and $\Sigma^{*} / S$ for semi-Thue systems $R$ and $S$, then the word problem of $\mathcal{M}$ with respect to $R$ is logspace-reducible to the word problem of $\mathcal{M}$ with respect to $S$. Thus, it makes sense to speak just of the word problem of $\mathcal{M}$ since we are only interested in the decidability (resp. complexity) status of word problems. Complexity results for monoids that are presented by various subclasses of semi-Thue systems can be found for instance in $[1,5,6,28,29]$.

The semi-Thue system $R$ is terminating if there does not exist an infinite chain $s_{1} \rightarrow_{R} s_{2} \rightarrow_{R} s_{3} \rightarrow_{R} \cdots$ in $\Gamma^{*}$. The set of irreducible words with respect to $R$ is $\operatorname{IRR}(R)=\left\{s \in \Gamma^{*} \mid \neg \exists t \in \Gamma^{*}: s \rightarrow_{R} t\right\}$. The system $R$ is confluent (resp. locally confluent) if for all $s, t, u \in \Gamma^{*}$ with $s \stackrel{*}{\rightarrow}_{R} t$ and $s \stackrel{*}{\rightarrow}_{R} u$ (resp. $s \rightarrow_{R} t$ and $s \rightarrow_{R} u$ ) there exists $w \in \Gamma^{*}$ with $t \stackrel{*}{\rightarrow}_{R} w$ and $u \stackrel{*}{\rightarrow}_{R} w$. If $R$ is terminating, then by Newman's lemma $R$ is confluent if and only if $R$ is locally confluent. Using critical pairs [7] which result from overlapping left-hand sides of $R$, local confluence is decidable for finite terminating semi-Thue systems. The system $R$ is length-reducing if $|s|>|t|$ for all $(s, t) \in R$, where $|w|$ is the length of a word $w$. The system $R$ is called length-lexicographic if there exists a linear order $\succ$ on the alphabet $\Gamma$ such that for every rule $(s, t) \in R$ either $|s|>|t|$ or $\left(|s|=|t|\right.$ and there are $u, v, w \in \Gamma^{*}$ and $a, b \in \Gamma$ such that $s=u a v, t=u b w$, and $a \succ b)$. Clearly, every lengthlexicographic semi-Thue system is terminating. In the case when $R$ is terminating and confluent, then every word $s$ has a unique normal form $\operatorname{NF}_{R}(s) \in \operatorname{IRR}(R)$ such that $s \stackrel{*^{*}}{\rightarrow} \mathrm{NF}_{R}(s)$ and moreover, the function $\pi_{R} \mid \operatorname{IRR}(R)$ (i.e., $\pi_{R}$ restricted to $\operatorname{IRR}(R))$ is bijective. Thus, if $R$ is in addition finite, then the word problem of $\Gamma^{*} / R$ is decidable: $\pi_{R}(s)=\pi_{R}(t)$ if and only if $\mathrm{NF}_{R}(s)=\mathrm{NF}_{R}(t)$.

\section{Automatic Monoids}

Automatic monoids were investigated for instance in [10, 18, 20, 22, 36]. They generalize automatic groups, see [15]. Let us fix a finite alphabet $\Gamma$. Let $\# \notin \Gamma$ be an additional padding symbol and let $\Gamma_{\#}=\Gamma \cup\{\#\}$. We define two encodings $\nu_{\ell}, \nu_{r}: \Gamma^{*} \times \Gamma^{*} \rightarrow\left(\Gamma_{\#} \times \Gamma_{\#}\right)^{*}$ as follows: Let $u, v \in \Gamma^{*}$ and let $k=\max \{|u|,|v|\}$. 
Define $w=u \#^{k-|u|}, x=v \#^{k-|v|}, y=\#^{k-|u|} u$, and $z=\#^{k-|v|} v$. Let $w[i]$ denote the $i$-th symbol of $w$ and similarly for $x, y$, and $z$. Then

$$
\nu_{r}(u, v)=(w[1], x[1]) \cdots(w[k], x[k]) \text { and } \nu_{\ell}(u, v)=(y[1], z[1]) \cdots(y[k], z[k]) .
$$

For instance,

$$
\begin{aligned}
& \nu_{r}(a b a, b b a b b)=(a, b)(b, b)(a, a)(\#, b)(\#, b) \text { and } \\
& \nu_{\ell}(a b a, b b a b b)=(\#, b)(\#, b)(a, a)(b, b)(a, b) .
\end{aligned}
$$

In the following let $\alpha, \beta \in\{\ell, r\}$. A relation $R \subseteq \Gamma^{*} \times \Gamma^{*}$ is called $\alpha$-automatic if the language $\left\{\nu_{\alpha}(u, v) \mid(u, v) \in R\right\}$ is a regular language over the alphabet $\Gamma_{\#} \times \Gamma_{\#}$. A relation $R \subseteq \Gamma^{*} \times \Gamma^{*}$ has bounded length-difference if there exists a constant $\gamma$ such that for all $(u, v) \in R,|(|u|-|v|)| \leq \gamma$. The following simple lemma will turn out to be useful. Its simple proof is left to the reader.

Lemma 1 Let $R, S \subseteq \Gamma^{*} \times \Gamma^{*}$ have bounded length-difference. Then $R$ is $\ell$ automatic if and only if $R$ is $r$-automatic. Moreover, if $R$ and $S$ are $\alpha$-automatic, then $R \cdot S=\{(s t, u v) \mid(s, u) \in R,(t, v) \in S\}$ is $\alpha$-automatic as well.

Let $\mathcal{M}$ be a monoid. A triple $(\Gamma, R, L)$ is an $\alpha \beta$-automatic presentation for $\mathcal{M}$ if: (i) $R$ is a semi-Thue system over the finite alphabet $\Gamma$ such that $\mathcal{M} \simeq$ $\Gamma^{*} / R$, (ii) $L \subseteq \Gamma^{*}$ is a regular language such that $\pi_{R}\lceil L$ maps $L$ surjectively to $\mathcal{M}$, (iii) the relation $\left\{(u, v) \in L \times L \mid \pi_{R}(u)=\pi_{R}(v)\right\}$ is $\alpha$-automatic, and (iv) if $\beta=\ell$ (resp. $\beta=r$ ), then the relation $\left\{(u, v) \in L \times L \mid \pi_{R}(a u)=\pi_{R}(v)\right\}$ (resp. $\left.\left\{(u, v) \in L \times L \mid \pi_{R}(u a)=\pi_{R}(v)\right\}\right)$ is $\alpha$-automatic for every $a \in \Gamma$. Let $T \subseteq\{\ell \ell, \ell r, r \ell, r r\}$ with $T \neq \emptyset$. Then the monoid $\mathcal{M}$ is $T$-automatic if there exists a triple $(\Gamma, R, L)$, which is a $t$-automatic presentation for $\mathcal{M}$ for all $t \in T$ (thus, by definition $\mathcal{M}$ is finitely generated). For $t \in\{\ell \ell, \ell r, r \ell, r r\}$ we write $t$-automatic instead of $\{t\}$-automatic. Usually, $r r$-automaticity (i.e., padding and multiplication on the right) is meant, when only the term automatic is used. If $\mathcal{M}$ is $\{r \ell, r r\}$ automatic, then $\mathcal{M}$ is also called biautomatic. In $[18,20]$, it was shown that for all $T_{1}, T_{2} \subseteq\{\ell \ell, \ell r, r \ell, r r\}$ with $\emptyset \neq T_{1} \neq T_{2} \neq \emptyset$ there exists a $T_{1}$-automatic monoid, which is not $T_{2}$-automatic. Thus, for monoids there are 15 different notions of automaticity. For groups, the situation is different. For instance, it is not hard to see that for $t_{1}, t_{2} \in\{\ell \ell, \ell r, r \ell, r r\}$ with $t_{1} \neq t_{2}$, a group is $t_{1}$-automatic if and only if it is $t_{2}$-automatic. On the other hand, it is open whether every $(r r$-)automatic group is already biautomatic. For our lower bounds we will mostly work with the strongest possible notion of automaticity, i.e., $\{\ell \ell, \ell r, r \ell, r r\}$-automaticity. Various classes of semi-Thue systems that present automatic monoids can be found in [36].

\section{Complexity of the Word Problem}

The word problem for an automatic group can be solved in quadratic time [15]. Moreover, the same algorithm also works for $\alpha \beta$-automatic monoids [10]. Here we will show that $\mathrm{P}$ is also a lower bound for the monoid case.

Theorem 1 There is a finite, length-lexicographic, and confluent semi-Thue system $R \subseteq \Gamma^{*} \times \Gamma^{*}$ such that the word problem for $\Gamma^{*} / R$ is $P$-complete and $(\Gamma, R, \operatorname{IRR}(R))$ is an $\alpha \beta$-automatic presentation for $\Gamma^{*} / R$ for all $\alpha, \beta \in\{\ell, r\}$. 
Proof. We start with a fixed deterministic Turing machine $S$ that accepts a P-complete language. Let $p(n)$ be a polynomial such that $S$ terminates on an input $w \in L(S)$ after exactly $p(|w|)$ steps; this exact time bound can be easily enforced. We may assume that the tape is restricted to size $p(|w|)$. It is straight forward to simulate $S$ by a new deterministic Turing machine $T$ that operates in a sequence of complete left/right sweeps over the whole tape (of size $p(|w|)$ ). During a right sweep, the head runs from the left tape end to the right tape end in a sequence of right moves. When reaching the right tape end, the head turns back and starts a left sweep. Let $\Sigma$ be the tape alphabet of $T, Q$ be the set of states, $q_{0}$ be the initial state, and $q_{f}$ be the final state. With $\phi \in \Sigma$ we denote the blank symbol. We write $q a \Rightarrow_{T} b p\left(a q \Rightarrow_{T} p b\right)$, in case $T$ writes $b$, moves right (left), and enters state $p$, when reading $a$ in state $q$. The machine $T$ terminates (and accepts its input) if and only if it finally reaches the final state $q_{f}$. Thus, $T$ cannot make any transitions out of $q_{f}$. Moreover, we may assume that the tape is blank and that the tape head is scanning the first cell when $T$ terminates in state $q_{f}$. Define $\Gamma=\Sigma \cup \bar{\Sigma} \cup Q \cup \bar{Q} \cup\{\$, \$\}$, where $\bar{\Sigma}=\{\bar{a} \mid a \in \Sigma\}$ is a disjoint copy of $\Sigma$ and similarly for $\bar{Q}$. Let $R$ be the following semi-Thue system over $\Gamma$ :

$$
\begin{array}{llll}
q a \rightarrow \bar{b} p & \text { if } q a \Rightarrow_{T} b p & \bar{a} \bar{q} \rightarrow \bar{p} b & \text { if } a q \Rightarrow_{T} p b \\
q \$ \rightarrow \bar{q} & \text { for all } q \in Q & \$ \bar{q} \rightarrow q & \text { for all } q \in Q
\end{array}
$$

$R$ is length-lexicographic and confluent (there are no critical pairs since $T$ is deterministic). Next, let $w \in \Sigma^{*}$ be an arbitrary input for $T$ and let $m=p(|w|)$. Then $w$ is accepted by $T$ if and only if $\overline{\$}^{m} q_{0} w \mathbb{c}^{m-|w|} \$^{m} \stackrel{*}{\rightarrow}_{R} q_{f} \mathbb{C}^{m}$ if and only if $\overline{\$}^{m} q_{0} w \mathbb{c}^{m-|w|} \$^{m} \stackrel{*}{\longleftrightarrow} R q_{f} \mathbb{\Phi}^{m}$. Thus, the word problem for $\Gamma^{*} / R$ is P-hard.

Next, we show that for all $\alpha, \beta \in\{\ell, r\},(\Gamma, R, \operatorname{IRR}(R))$ is an $\alpha \beta$-automatic presentation for $\Gamma^{*} / R$ (then in particular, the word problem for $\Gamma^{*} / R$ belongs to $\mathrm{P}$ ). Due to the symmetry of $R$, we can restrict to $\beta=\ell$. Thus, we have to show that the relation $E_{c}=\left\{(u, v) \in \operatorname{IRR}(R) \times \operatorname{IRR}(R) \mid c u \stackrel{*}{\rightarrow}_{R} v\right\}$ is $\alpha$-automatic for all $c \in \Gamma$ and $\alpha \in\{\ell, r\}$. Note that all relations that appear in the following consideration have bounded length-difference. This allows to make use of Lemma 1. First, note that the following relations are $\alpha$-automatic:

$$
\begin{aligned}
& A_{q}=\left\{(u, \bar{v} p) \mid p \in Q, u \in \Sigma^{*}, \bar{v} \in \bar{\Sigma}^{*}, q u \stackrel{*}{\rightarrow}_{R} \bar{v} p\right\} \\
& B_{q}=\left\{(\bar{u}, \bar{p} v) \mid \bar{p} \in \bar{Q}, \bar{u} \in \bar{\Sigma}^{*}, v \in \Sigma^{*}, \bar{u} \bar{q} \stackrel{*}{\rightarrow}_{R} \bar{p} v\right\}
\end{aligned}
$$

The relation $A_{q}$ (resp. $B_{q}$ ) describes a single right (resp. left) sweep over the whole tape started in state $q$, which is just a rational transduction. Since $\alpha$-automatic relations are closed under composition, the relation

$$
C_{q}=\left\{(u \$, \bar{p} v) \mid \bar{p} \in \bar{Q}, u, v \in \Sigma^{*}, q u \$ \stackrel{*}{\rightarrow}_{R} \bar{p} v\right\}
$$

is $\alpha$-automatic as well. Now the $\alpha$-automaticity of the relations $E_{c}$ for $c \in \Gamma$ follows easily: For $c \in \bar{Q} \cup \Sigma \cup\{\$\}$ we have $E_{c}=\{(u, c u) \mid u \in \operatorname{IRR}(R)\}$, which is clearly 
$\alpha$-automatic. For $c=\bar{a} \in \bar{\Sigma}$ and $c=q \in Q$, respectively, we have:

$$
\begin{aligned}
E_{\bar{a}}= & \left\{(u, \bar{a} u) \mid u \in \operatorname{IRR}(R), u \notin \bar{Q} \Gamma^{*}\right\} \cup \\
& \{(\bar{q} u, \bar{p} b u) \mid u \in \operatorname{IRR}(R), \bar{q}, \bar{p} \in \bar{Q}, b \in \Sigma,(\bar{a} \bar{q}, \bar{p} b) \in R\} \\
E_{q}= & \left\{(u w, v w) \mid(u, v) \in A_{q}, w \in \operatorname{IRR}(R), w \notin(\Sigma \cup\{\$\}) \Gamma^{*}\right\} \cup \\
& \left\{(u w, v w) \mid(u, v) \in C_{q}, w \in \operatorname{IRR}(R)\right\} .
\end{aligned}
$$

Finally, $E_{\bar{\Phi}}=\left\{(u, \overline{\$} u) \mid u \in \operatorname{IRR}(R), u \notin \bar{Q} \Gamma^{*}\right\} \cup \bigcup_{q \in Q}\left\{(\bar{q} u, v) \mid(u, v) \in E_{q}\right\}$. This concludes the proof of the $\alpha$-automaticity of the relations $E_{c}$.

Corollary 1 There exists a fixed finitely presented $\{\ell \ell, \ell r, r \ell, r r\}$-automatic monoid with a P-complete word problem.

It is open, whether there exists an automatic group (or at least a cancellative automatic monoid) with a P-complete word problem. An important subclass of the class of automatic groups is the class of hyperbolic groups, which are defined via a geometric hyperbolicity condition on the Cayley-graph [16]. The precise definition is not important for the purpose of this paper. In [8], Cai has shown that for every hyperbolic group the word problem belongs to the parallel complexity class $\mathrm{NC}^{2}$, which is the class of all problems that can be recognized by a polynomial size family of Boolean circuits of depth $\mathcal{O}\left(\log ^{2}(n)\right)$, where only Boolean gates of fan-in at most 2 are allowed. Cai also asked, whether the upper bound of $\mathrm{NC}^{2}$ can be improved. Using a known result from formal language theory, we will show that for every hyperbolic group the word problem belongs to LOGCFL $\subseteq \mathrm{NC}^{2}$, which is the class of all problems that are logspace reducible to a context-free language [44]. For alternative characterizations of LOGCFL see [39, 45].

Theorem 2 The word problem for every fixed hyperbolic group is in LOGCFL.

Proof. By [12], a group $\mathcal{G}$ is hyperbolic if and only if $\mathcal{G} \cong \Gamma^{*} / R$, where $R$ is finite, length-reducing, and $\left\{s \in \Gamma^{*} \mid s \stackrel{*}{\rightarrow}_{R} \varepsilon\right\}=\left\{s \in \Gamma^{*} \mid s \stackrel{*}{\leftrightarrow}_{R} \varepsilon\right\}$. Let $L$ be the latter language. Since $\mathcal{G}$ is a group, the word problem for $\mathcal{G}$ is logspace reducible to $L$. Since $R$ is length-reducing, $L$ is growing context-sensitive, i.e., it can be generated by a grammar, where every production is strictly length-increasing. Since every fixed growing context-sensitive language belongs to LOGCFL [13], the theorem follows.

In $[14,19]$, hyperbolic groups were generalized to hyperbolic monoids. It is not clear whether Theorem 2 can be extended to hyperbolic monoids. It is also open, whether the upper bound of LOGCFL from Theorem 2 can be further improved, for instance to LOGDCFL, which is the class of all problems that are logspace reducible to a deterministic context-free language [44]. For another class of automatic monoids, we can precisely characterize the complexity of the word problem using LOGDCFL: A semi-Thue system $R$ over the alphabet $\Gamma$ is called left-basic [41] if: (i) if $\ell \in \operatorname{dom}(R), r \in \operatorname{ran}(R)$ and $r=u \ell v$ then $u=v=\varepsilon$ and (ii) if $\ell \in \operatorname{dom}(R)$, $r \in \operatorname{ran}(R)$, ur $=\ell v$, and $|\ell|>|u|$, then $v=\varepsilon$. Condition (i) means that a righthand side does not strictly contain a left-hand side. Condition (ii) means that the following kind of overlapping is not allowed: 


\begin{tabular}{|c|c|c|}
\hline$u$ & \multicolumn{2}{|c|}{$r \in \operatorname{ran}(R)$} \\
\hline \multicolumn{2}{|c|}{$\ell \in \operatorname{dom}(R)$} & $v \neq \varepsilon$ \\
\hline
\end{tabular}

Let us define the suffix-rewrite relation $\rightarrow_{R}$ by $s \rightarrow_{R} t$ if and only if $s=u \ell$ and $t=u r$ for some $u \in \Gamma^{*}$ and $(\ell, r) \in R$. The following lemma is obvious:

Lemma 2 If $R$ is left-basic, then for every $s \in \operatorname{IRR}(R)$ and $a \in \Gamma$ we have $s a \stackrel{*}{\rightarrow}_{R} t$ if and only if $s a \stackrel{*}{\rightarrow} R$ t.

Left-basic semi-Thue systems generalize monadic semi-Thue systems (where $|\ell|>|r| \leq 1$ for every rule $\ell \rightarrow r)$. Systems that are finite, monadic, and confluent present monoids that are simultaneously $r r$ - and $\ell \ell$-automatic, but in general neither $r \ell$ - nor $\ell r$-automatic [36]. For left-basic systems we obtain the following result:

Proposition 1 If $R$ is a terminating, confluent, and left-basic semi-Thue system over the alphabet $\Gamma$, then $\Gamma^{*} / R$ is rr-automatic.

Proof. Let $R$ be terminating, confluent, and left-basic. We define the semiThue system $S$ by $S=\left\{(s, t) \mid \exists(\ell, r) \in R: \ell=s \wedge t=\mathrm{NF}_{R}(r)\right\}$. Since $S$ is left-basic, we have $\operatorname{ran}(S) \subseteq \operatorname{ran}(R)$. Since moreover $\operatorname{dom}(S)=\operatorname{dom}(R)$, the system $S$ is still left-basic. By [7, Lemma 2.2.11], $S$ is also terminating and confluent, and $\Gamma^{*} / R \cong \Gamma^{*} / S$. We claim that $(\Gamma, S, \operatorname{IRR}(S))$ is an $r r$-automatic presentation for $\Gamma^{*} / S$. For this, it suffices to prove the following claim, which implies that $\left\{(s, t) \in \operatorname{IRR}(S) \times \operatorname{IRR}(S) \mid s a \stackrel{*}{\rightarrow}_{S} t\right\}$ is $r$-automatic for every $a \in \Gamma$.

Claim: Let $s \in \operatorname{IRR}(S)=\operatorname{IRR}(R)$ and $a \in \Gamma$. Then, $s a \stackrel{*}{\rightarrow}_{S} t$ if and only if $s a=t$ or there exist $u \in \operatorname{IRR}(S), k \geq 0$, rules $\left(\ell_{i}, r_{i}\right) \in S$ for $0 \leq i \leq k$, and words $\ell_{i}^{\prime} \in \Gamma^{*} \backslash\{\varepsilon\}$ such that $\ell_{i}=\ell_{i}^{\prime} r_{i-1}(1 \leq i \leq k), s a=u \ell_{k}^{\prime} \ell_{k-1}^{\prime} \cdots \ell_{1}^{\prime} \ell_{0}$, and $t=u r_{k}$.

The "if" direction in this claim is obvious. For the "only if"-direction let us take $s \in \operatorname{IRR}(S)$ and $a \in \Gamma$ and assume that $s a \stackrel{*}{\rightarrow}_{S} t$. If we do not have $s a=t$, then there exists some $s^{\prime}$ with $s a \rightarrow_{S} s^{\prime} \stackrel{*}{\rightarrow}_{S} t$. Lemma 2 implies that $s a=s_{0} \ell_{0}$ and $s^{\prime}=s_{0} r_{0} \stackrel{*}{\rightarrow}_{S} t$ for some $s_{0} \in \operatorname{IRR}(S)$ and some rule $\left(\ell_{0}, r_{0}\right) \in S$. We now prove by induction on the length of the derivation $s_{0} r_{0} \stackrel{*}{\rightarrow}_{S} t$ that there exist $u \in \operatorname{IRR}(S)$, $k \geq 0$, rules $\left(\ell_{i}, r_{i}\right) \in S$ and words $\ell_{i}^{\prime} \in \Gamma^{*} \backslash\{\varepsilon\}(1 \leq i \leq k)$ such that $\ell_{i}=\ell_{i}^{\prime} r_{i-1}$ $(1 \leq i \leq k), s_{0}=u \ell_{k}^{\prime} \ell_{k-1}^{\prime} \cdots \ell_{1}^{\prime}$, and $t=u r_{k}$, which implies the claim. If we have $s_{0} r_{0}=t$, then we choose $k=0$ and $u=s_{0}$. Otherwise, we have $s_{0} r_{0} \rightarrow_{S} s_{0}^{\prime} \stackrel{*}{\rightarrow}_{S} t$ for some $s_{0}^{\prime}$. Since $S$ is left-basic and $r_{0} \in \operatorname{ran}(S) \subseteq \operatorname{IRR}(S)$, there exists a rule $\left(\ell_{1}, r_{1}\right) \in S$ and a word $\ell_{1}^{\prime} \in \Gamma^{*} \backslash\{\varepsilon\}$ such that $\ell_{1}=\ell_{1}^{\prime} r_{0}, s_{0}=s_{1} \ell_{1}^{\prime}$ for some $s_{1} \in \operatorname{IRR}(S)$, and $s_{0}^{\prime}=s_{1} r_{1} \stackrel{*}{\rightarrow} S$. Now we can conclude by induction.

Theorem 3 The following problem is in LOGDCFL:

INPUT: A finite, terminating, confluent, and left-basic semi-Thue system $R$ over an alphabet $\Gamma$, and two words $s, t \in \Gamma^{*}$

QUESTION: $s \stackrel{*}{\leftrightarrow} R t$ ?

Moreover, there exists a finite, length-reducing, confluent, and left-basic semi-Thue system $R$ over an alphabet $\Gamma$ such that the word problem for $\Gamma^{*} / R$ is LOGDCFLcomplete.

Proof. Note that the upper bound in the first statement holds in a uniform setting, i.e., the semi-Thue system is part of the input. In order to prove this upper 
bound, we will use a machine-based characterization of LOGDCFL: A logspace bounded deterministic AuxPDA is a deterministic pushdown automaton that has an auxiliary read-write tape of size $\mathcal{O}(\log (n))$ (where $n$ is the input size). A problem belongs to LOGDCFL if and only if it can be decided by a logspace bounded deterministic AuxPDA that moreover works in polynomial time [44].

Now, let us assume that the input consists of a tuple $(\Gamma, R, s, t)$, where $R$ is a finite, terminating, confluent, and left-basic semi-Thue system over the alphabet $\Gamma$ and $s, t \in \Gamma^{*}$. Let $n$ be the length of the binary coding of this input. We will construct a logspace bounded deterministic AuxPDA that checks in polynomial time, whether $\mathrm{NF}_{R}(s)=\mathrm{NF}_{R}(t)$. For this, we will first show how to calculate $\mathrm{NF}_{R}(s)$ on a deterministic AuxPDA in logspace and polynomial time. The basic idea of how to do this appeared many times in the literature, see e.g. [7, Thm. 4.2.7]. The only slight complication in our situation results from the fact that the semiThue system $R$ belongs to the input. To overcome this, we need the logspace bounded auxiliary store of our AuxPDA. The correctness of the following procedure follows from Lemma 2. Our algorithm for computing $\mathrm{NF}_{R}(s)$ works in stages. At the beginning of a stage the pushdown contains a word from $\operatorname{IRR}(R)$ and the auxiliary store contains a pointer to a position $i$ in the input word $s$. Note that a symbol $a \in \Gamma$ can be represented as a bit string of length $\mathcal{O}(\log (n))$, thus the pushdown content is a sequence of blocks of length $\mathcal{O}(\log (n))$, where every block represents a symbol from $\Gamma$. The stage begins by pushing the $i$-th symbol of $s$ onto the pushdown (which is a bit string of length $\mathcal{O}(\log (n))$ ) and incrementing the pointer to position $i+1$ in $s$. Now we have to check whether the pushdown content is from $\Gamma^{*} \operatorname{dom}(R)$. For this we have to scan every left-hand side of $R$ using a second pointer to the input. Every $\ell \in \operatorname{dom}(R)$ is scanned in reverse order and thereby compared with the top of the push-down. During this phase, symbols are popped from the pushdown. If it turns out that the left-hand side that is currently scanned is not a suffix of the pushdown content, then these symbols must be "repushed". This can be done, since the suffix of the pushdown content that was popped so far is a suffix of the currently scanned left-hand side $\ell \in \operatorname{dom}(R)$, which is still available on the read-only input tape. If a left-hand side $\ell$ is found on top of the pushdown, then the corresponding right-hand side is pushed on the pushdown and we try to find again a left-hand side on top of the pushdown. If finally no left-hand side matches a suffix of the pushdown content, then we know that the pushdown content belongs to $\operatorname{IRR}(R)$ and we can proceed with the next stage. Finally, if the first pointer has reached the end of the input word $s$ (or more precisely points to the first position following $s$ ), then the pushdown content equals $\mathrm{NF}_{R}(s)$.

Claim: In the above procedure, after the $i$-th stage the pushdown has length at most $i \cdot \alpha$, where $\alpha=\max (\{1\} \cup\{|r| \mid r \in \operatorname{ran}(R)\})$. Moreover, every stage needs only polynomial time.

The first statement can be shown by induction on $i$. Since $R$ is left-basic, it follows that if $w$ is the pushdown content at the end of the $(i-1)$-th stage, then the pushdown content at the end of the $i$-th stage either belongs to $w \Gamma$ or is of the form $u r$ for some $r \in \operatorname{ran}(R)$ and some prefix $u$ of $w$. Moreover, since $R$ is terminating 
and left-basic, the $i$-th stage simulates at most $|w| \cdot|R|$ rewrite steps of $R$.

In order to check whether $\mathrm{NF}_{R}(s)=\mathrm{NF}_{R}(t)$, we have to solve one more problem: If we would calculate $\mathrm{NF}_{R}(t)$ in the same way as above, then the pushdown would finally contain the word $\mathrm{NF}_{R}(s) \mathrm{NF}_{R}(t)$. But now there seems to be no way of checking, whether $\mathrm{NF}_{R}(s)=\mathrm{NF}_{R}(t)$. Thus, we have to apply another strategy. Note that for a fixed binary coded number $1 \leq i \leq \alpha \cdot|s|$, it is easy to modify our algorithm for calculating $\mathrm{NF}_{R}(s)$ such that some specified auxiliary storage cell $S$ contains always the $i$-th symbol of the pushdown content (or some special symbol if the pushdown is shorter than $i$ ). For this we have to store the current length of the pushdown, for which we need only space $\mathcal{O}(\log (n))$. Moreover, also $S$ only needs space $\mathcal{O}(\log (n))$. Thus, at the end of our modified algorithm for computing $\mathrm{NF}_{R}(s)$, $S$ contains the symbol $\mathrm{NF}_{R}(s)[i]$ (the $i$-th symbol of $\mathrm{NF}_{R}(s)$ ) or some special symbol in case $\left|\mathrm{NF}_{R}(s)\right|<i$. Next, we flush the pushdown and repeat the same procedure with the other input word $t$ and the same $i$, using another storage cell $T$. In this way we can check, whether $\mathrm{NF}_{R}(s)[i]=\mathrm{NF}_{R}(t)[i]$. Finally, we repeat this step for every $1 \leq i \leq \max \{\alpha \cdot|s|, \alpha \cdot|t|\}$. The latter bound is the maximal pushdown-length that may occur, which follows from the above claim. Note that also $i$ needs only space $\mathcal{O}(\log (n))$. This concludes the description of our LOGDCFL-algorithm.

It remains to construct a finite, length-reducing, confluent, and left-basic semiThue system $R$ such that the corresponding word problem is LOGDCFL-hard. In [44], Sudborough has shown that there exists a fixed deterministic context-free language $L \subseteq \Sigma^{*}$ with a LOGDCFL-complete membership problem. Let $\mathcal{A}=$ $\left(Q, \Delta, \Sigma, \delta, q_{0}, \perp\right)$ be a deterministic pushdown automaton with $L=L(\mathcal{A})$, where $Q$ is the set of states, $q_{0} \in Q$ is the initial state, $\Delta$ is the pushdown alphabet, $\perp \in \Delta$ is the bottom symbol, and $\delta: \Delta \times Q \times \Sigma \rightarrow \Delta^{*} \times Q$ is the transition function. By [44, Lem. 7] we may assume that $\mathcal{A}$ makes no $\varepsilon$-moves and that $\mathcal{A}$ accepts $L$ by empty store in state $q_{0}$. Let $m=\max \{|\gamma| \mid \delta(A, q, a)=(\gamma, p), q, p \in Q, A \in \Delta, a \in \Sigma\}$; thus, $m$ is the maximal length of a sequence that is pushed on the pushdown in one step. Let $\# \notin \Delta \cup Q \cup \Sigma$ be an additional symbol and let $\Gamma=\Delta \cup Q \cup \Sigma \cup\{\#\}$. Define the semi-Thue system $R$ by $R=\left\{A q a^{m} \# \rightarrow \gamma p \mid \delta(A, q, a)=(\gamma, p)\right\}$; it is length-reducing, confluent, and left-basic. Moreover, if $h: \Sigma^{*} \rightarrow(\Sigma \cup\{\#\})^{*}$ denotes the homomorphism defined by $h(a)=a^{m} \#$, which can be computed in logspace, then $w \in L$ if and only if $\perp q_{0} h(w) \stackrel{*}{\rightarrow}_{R} q_{0}$ if and only if $\perp q_{0} h(w) \stackrel{*}{\leftrightarrow}_{R} q_{0}$.

\section{Cayley-graphs}

Let $\mathcal{M}=(M, \circ, 1)$ be a monoid, which is finitely generated by $\Gamma$. The right Cayley-graph of $\mathcal{M}$ with respect to $\Gamma$ is the $\Gamma$-labeled rooted directed graph

$$
\mathcal{C}(\mathcal{M}, \Gamma)=\left(M,(\{(u, v) \mid u \circ a=v\})_{a \in \Gamma}, 1\right) .
$$

Thus, edges are defined via multiplication with generators on the right and the neutral element 1 is added as a constant (the root). The graph that is defined analogously via multiplication with generators on the left is called the left Cayley-graph of $\mathcal{M}$ with respect to $\Gamma$. In the following, we will always refer to the right Cayley-graph when just speaking of the Cayley-graph. Cayley-graphs were mainly investigated 
for groups, in particular they play an important role in combinatorial group theory [32] (see also the survey of Schupp [40]). Combinatorial properties of Cayley-graphs of monoids are studied in [23, 46]. In [42, 43], Cayley-graphs of automatic monoids are investigated. The work of Calbrix and Knapik on Thue-specifications [9, 25] covers Cayley-graphs of monoids that are presented by terminating and confluent semi-Thue systems as a special case.

In $[26,27]$, an investigation of Cayley-graphs from a logical point of view was initiated. For a given Cayley-graph $\mathcal{C}=\left(M,\left(E_{a}\right)_{a \in \Gamma}, 1\right)$ we consider first-order formulas over the structure $\mathcal{C}$. Atomic formulas are of the form $x=y, x=1$, and $E_{a}(x, y)$, (there is an $a$-labeled edge from $x$ to $y$ ) where $x$ and $y$ are variables that range over $M$. Instead of $(x, y) \in E_{a}$ we write $x \circ a=y$, or briefly $x a=y$. First-order formulas are built from atomic formulas using Boolean connectives and quantifications over variables. The notion of a free variable is defined as usual. A first-order formula without free variables is called a first-order sentence. For a first-order sentence $\varphi$, we write $\mathcal{C} \models \varphi$ if $\varphi$ evaluates to true in $\mathcal{C}$. The first-order theory of the Cayley-graph $\mathcal{C}$, denoted by $\operatorname{FOTh}(\mathcal{C})$, is the set of all first-order sentences $\varphi$ such that $\mathcal{C} \models \varphi$. We will also use more general atomic formulas of the form $x u=y$ and $x u=y v$ for variables $x, y$ and words $u, v \in \Gamma^{*}$, with the obvious interpretation. These new atomic formulas can be easily eliminated using fresh existentially quantified variables. For a detailed introduction into first-order logic over arbitrary structures see [17].

Many interesting monoid properties can be expressed using first-order logic over the Cayley-graph. For instance the following first-order sentence expresses that the monoid $\mathcal{M}$ (with generating set $\Gamma$ ) is right-cancellative:

$$
\bigwedge_{a \in \Gamma} \forall x \forall y\{x a=y a \rightarrow x=y\}
$$

If the monoid $\mathcal{M}$ is finitely generated both by $\Gamma$ and $\Sigma$, then $\operatorname{FOTh}(\mathcal{C}(\mathcal{M}, \Gamma))$ is logspace reducible to $\operatorname{FOTh}(\mathcal{C}(\mathcal{M}, \Sigma))$ and vice versa [27]. Thus, analogously to word problems, the decidability (resp. complexity) status of the first-order theory of a Cayley-graph does not depend on the chosen set of generators, and with respect to decidability and complexity questions we can just speak of the Cayley-graph of $\mathcal{M}$. From the definition of an $\alpha r$-automatic monoid $\mathcal{M}$ it follows immediately that $\mathcal{C}(\mathcal{M}, \Gamma)$ is an automatic graph in the sense of $[3,24]$ (but the converse is even false for groups, see e.g. [4]). Thus, since every automatic graph has a decidable firstorder theory [24], $\operatorname{FOTh}(\mathcal{C}(\mathcal{M}, \Gamma))$ is decidable in case $\mathcal{M}$ is $\alpha r$-automatic $(\alpha=\ell$ or $\alpha=r)$. If $\mathcal{M}$ is an $\alpha$-automatic monoid $(\alpha=\ell$ or $\alpha=r)$, then the first-order theory of the left Cayley-graph of $\mathcal{M}$ is decidable.

A problem is elementarily decidable if it can be solved in time $\mathcal{O}\left(2^{.2^{2}}\right)$, where the height of this tower of exponents is constant. By [3], there exists an automatic graph with a nonelementary first-order theory. This complexity is already realized by Cayley-graphs of automatic monoids:

Theorem 4 There is a finite, length-lexicographic, and confluent semi-Thue system $R \subseteq \Gamma^{*} \times \Gamma^{*}$ such that $(\Gamma, R, \operatorname{IRR}(R))$ is an $\alpha \beta$-automatic presentation for $\Gamma^{*} / R$ 
for all $\alpha, \beta \in\{\ell, r\}$ and $\operatorname{FOTh}\left(\mathcal{C}\left(\Gamma^{*} / R, \Gamma\right)\right)$ is nonelementary.

Proof. Let $\Gamma=\left\{a, b, \bar{a}, \bar{b}, \$_{1}, \$_{2}, \$_{a}\right\}$ and let the semi-Thue system $R$ over $\Gamma$ consist of the following rules, where $c \in\{a, b\}$ are arbitrary:

\begin{tabular}{llll|}
\hline$c \$_{1} \rightarrow \$_{1} c$ & $c \$_{2} \rightarrow \$_{2} c$ & $\bar{a} \$_{a} \rightarrow a$ & $c \$_{a} \rightarrow \$_{a} c$ \\
$\bar{c} \$_{1} \rightarrow c$ & $\bar{c} \$_{2} \rightarrow \$_{1} \bar{c}$ & $\bar{b} \$_{a} \rightarrow \$_{a} \bar{b}$ &
\end{tabular}

$R$ is length-lexicographic and confluent. Arguments similar to those from the proof of Theorem 1 show that $(\Gamma, R, \operatorname{IRR}(R))$ is an $\alpha \beta$-automatic presentation of $\mathcal{M}=\Gamma^{*} / R$. Let $\mathcal{C}=\mathcal{C}(\mathcal{M}, \Gamma)$. It remains to show that $\operatorname{FOTh}(\mathcal{C})$ is not elementarily decidable. For this we reduce the first-order theory of finite words over $\{a, b\}$ to $\operatorname{FOTh}(\mathcal{C})$. The former theory is defined as follows: A word $w=a_{1} a_{2} \cdots a_{n} \in\{a, b\}^{*}$ of length $n$ is identified with the relational structure $S_{w}=\left(\{1, \ldots, n\},<, Q_{a}\right)$, where $<$ is the usual order on natural numbers and $Q_{a}$ is the unary predicate $\left\{i \in\{1, \ldots, n\} \mid a_{i}=a\right\}$. Then the first-order theory of finite words over $\{a, b\}$ consists of all first-order sentences $\phi$ that are built up from the atomic formulas $x<y$ and $Q_{a}(x)$ such that $S_{w} \models \phi$ for every word $w \in\{a, b\}^{*}$. It is known that the first-order theory of finite words is decidable but not elementary, see e.g. [11, Example 8.1] for a simplified proof and further references.

For our reduction first notice that $\operatorname{IRR}(R)=\left\{\$_{1}, \$_{2}, \$_{a}\right\}^{*}\{a, b, \bar{a}, \bar{b}\}^{*}$. Hence, the latter set can be identified with the monoid $\mathcal{M}$. For $x \in \operatorname{IRR}(R)$ we have $x \in$ $\left\{\$_{1}, \$_{2}, \$_{a}\right\}^{*}\{a, b\}^{*}$ if and only if $x \$_{2} \$_{1} \neq x \$_{1} \$_{1}$ in $\mathcal{M}$. This allows us to represent all words from $\{a, b\}^{*}$ in $\mathcal{C}$. The fact that a word $w \in\{a, b\}^{*}$ is represented by infinitely many nodes of $\mathcal{C}$, namely by all elements from $\left\{\$_{1}, \$_{2}, \$_{a}\right\}^{*} w$ does not cause any problems; it is only important that every word $w \in\{a, b\}^{*}$ is represented at least once. In the sequel let us fix $x=v w$ with $v \in\left\{\$_{1}, \$_{2}, \$_{a}\right\}^{*}$ and $w \in\{a, b\}^{*}$. The set of all positions within the word $w$ is in one-to-one correspondence with the set of all $y$ such that $y \$_{1}=x$ in $\mathcal{M}$ : the latter holds if and only if $\exists w_{1}, w_{2} \in\{a, b\}^{*} \exists c \in\{a, b\}$ : $w=w_{1} c w_{2}$ and $y=v w_{1} \bar{c} w_{2}$. Thus, we can quantify over positions of the word $w$ by quantifying in $\mathcal{C}$ over all those nodes $y$ such that $y \$_{1}=x$ in $\mathcal{M}$. Next, assume that $y=v w_{1} \bar{c} w_{2}$ and $w=w_{1} c w_{2}$, i.e., $y$ represents the position $\left|w_{1}\right|+1$ of $w$. Then $c=a$ if and only if $y \$_{a}=x$ in $\mathcal{M}$; thus we can express that a position is labeled with the symbol $a$. It remains to express that a position is smaller than another one. Assume that $y=v w_{1} \bar{c} w_{2}, y^{\prime}=v w_{1}^{\prime} \bar{d} w_{2}^{\prime}, w_{1} c w_{2}=w_{1}^{\prime} d w_{2}^{\prime}=w$, and $w_{1} \neq w_{1}^{\prime}$, i.e., the two positions represented by $y$ and $y^{\prime}$ are different. Then $\left|w_{1}\right|<\left|w_{1}^{\prime}\right|$ if and only if $\exists z \in \mathcal{M}: z \$_{1}=y \wedge z \$_{2}=y^{\prime}$ in $\mathcal{M}$.

From the preceding discussion it follows that for every first-order sentence $\psi$ over the signature $\left(<, Q_{a}\right)$ we can construct in polynomial time a first-order formula $\phi(x)$ over the Cayley-graph $\mathcal{C}$ such that $\psi$ belongs to the first-order theory of finite words if and only if $\mathcal{C} \models \forall x: \phi(x)$. This proves the theorem.

Corollary 2 There exists a finitely presented $\{\ell \ell, \ell r, r \ell, r r\}$-automatic monoid $\mathcal{M}$ such that $\operatorname{FOTh}(\mathcal{C}(\mathcal{M}, \Gamma))$ is not elementarily decidable.

In $[26]$ it was shown that for a group $\mathcal{G}, \operatorname{FOTh}(\mathcal{C}(\mathcal{G}))$ is elementarily reducible to the word problem for $\mathcal{G}$. Since the word problem of an automatic group can be 
solved in time $\mathcal{O}\left(n^{2}\right)$, it follows that the nonelementary lower bound from Corollary 2 cannot be realized by an automatic group. This fact even holds for automatic monoids of finite geometric type: A finitely generated monoid $\mathcal{M}$ has finite geometric type if for some (and hence every) finite generating set $\Gamma$, the (undirected version of the) Cayley-graph $\mathcal{C}(\mathcal{M}, \Gamma)$ has bounded degree [43], i.e., the number of neighbors of any node is bounded by a fixed constant. Every right-cancellative monoid has finite geometric type, but for instance the bicyclic monoid $\{a, b\}^{*} /\{(a b, \varepsilon)\}$ is not right-cancellative but has finite geometric type. Since the Cayley-graph of an $\alpha r$-automatic monoid of finite geometric type is an automatic graph of bounded degree, and the first-order theory of every automatic graph of bounded degree belongs to $\operatorname{DSPACE}\left(2^{2^{2^{\mathcal{O}(n)}}}\right)[30]$, we obtain:

Theorem 5 Let $\mathcal{M}$ be an $\alpha$-automatic monoid $(\alpha \in\{r, \ell\})$ of finite geometric type. Then $\operatorname{FOTh}(\mathcal{C}(\mathcal{M}, \Gamma))$ belongs to $\operatorname{DSPACE}\left(2^{2^{2^{\mathcal{O}(n)}}}\right)$ and thus is elementary.

We conclude this paper with an undecidability result for automatic monoids. Note that for an $\alpha r$-automatic monoid $\mathcal{M}(\alpha \in\{r, \ell\})$ it is decidable whether for given $u, v \in \mathcal{M}$ there exists $x \in \mathcal{M}$ such that $x u=v$ in $\mathcal{M}$, because this is a first-order property of the Cayley-graph. On the other hand, the reverse question ( $\exists x: u x=v$, i.e., reachability in the Cayley-graph) is undecidable in general:

Theorem 6 There exists a finitely presented $\{\ell r, r r\}$-automatic monoid $\mathcal{M}$ such that for given $u, v \in \mathcal{M}$ it is undecidable whether $\exists x \in \mathcal{M}: u x=v$ in $\mathcal{M}$.

Proof. Analogously to the proof of Theorem 1 we may start from a deterministic Turing machine $T$ with an undecidable acceptance problem that operates in a sequence of complete left/right sweeps. This time, we cannot work with a fixed size portion of the tape. Therefore, we assume that each time the tape head reaches the right end of the tape, an additional blank cell is added to the tape. This ensures that there is always enough space available. Let $Q$ be the state set of $T, \Sigma$ be the tape alphabet, $q_{0}$ be the initial state, and $q_{f}$ be the final state. $T$ cannot make any transitions out of the final state $q_{f}$ and the tape head points to the first cell when $T$ reaches $q_{f}$. Define $\Gamma=\Sigma \cup \bar{\Sigma} \cup Q \cup \bar{Q} \cup\{\triangleright, \triangleleft, \$\}$ and let $R$ be the following semi-Thue system over $\Gamma$ :

\begin{tabular}{|llll|}
\hline$q a \rightarrow \bar{b} p$ & if $q a \Rightarrow_{T} b p$ & $\bar{a} \bar{q} \rightarrow \bar{p} b$ & if $a q \Rightarrow_{T} p b$ \\
$q \triangleleft \$ \rightarrow \bar{q} \square \triangleleft$ & for all $q \in Q$ & $\triangleright \bar{q} \rightarrow \triangleright q$ & for all $q \in Q$ \\
$\triangleright q_{f} a \rightarrow \triangleright q_{f}$ & for all $a \in \Sigma$ & & \\
\hline
\end{tabular}

Then $R$ is length-lexicographic (when strings are compared from right to left), and by analyzing critical pairs, we see that $R$ is confluent. Thus $\Gamma^{*} / R$ is in one-toone correspondence with $\operatorname{IRR}(R)$. We claim that an input word $w \in \Sigma^{*}$ is accepted by $T$ if and only if there exists $x \in \Gamma^{*}$ such that $\triangleright q_{0} w \triangleleft x \stackrel{*}{\leftrightarrow}_{R} \triangleright q_{f} \triangleleft$. Clearly, if $w$ is accepted by $T$, then there exists $m \geq 0$ such that $\triangleright q_{0} w \triangleleft \$^{m} \stackrel{*}{\leftrightarrow} R \triangleright q_{f} \triangleleft$. On the other hand, assume that $\triangleright q_{0} w \triangleleft x \stackrel{*}{\leftrightarrow} R \triangleright q_{f} \triangleleft$, i.e., $\triangleright q_{0} w \triangleleft x \stackrel{*}{\rightarrow}_{R} \triangleright q_{f} \triangleleft$ for some $x \in \Gamma^{*}$. Let $x=\$^{m} y$ for some $m \geq 0$ and $y \notin \$ \Gamma^{*}$. In order to deduce a contradiction, assume that $T$ does not terminate on input $w$. Hence, for some state $q \neq q_{f}$ and $\bar{v} \in \bar{\Sigma}^{*}$ we have $\triangleright q_{0} w \triangleleft x=\triangleright q_{0} w \triangleleft \$^{m} y \stackrel{*}{\rightarrow}_{R} \triangleright \bar{v} q \triangleleft y \in \operatorname{IRR}(R)$. Since $R$ is confluent, this contradicts $\triangleright q_{0} w \triangleleft x \stackrel{*}{\rightarrow}_{R} \triangleright q_{f} \triangleleft \in \operatorname{IRR}(R)$. This proves the undecidability statement 
from the theorem. The $\alpha r$-automaticity $(\alpha=\ell$ and $\alpha=r$ ) of the presentation $(\Gamma, R, \operatorname{IRR}(R))$ can be shown as in the proof of Theorem 1 .

\section{Final remarks}

Many interesting open problems concerning the complexity of word problems remain:

- Is LOGCFL an optimal upper bound for the word problem of a hyperbolic group? Let us mention that the word problem of a hyperbolic group can be recognized in real time [21].

- What is the complexity of the word problem for monoids that can be presented by monadic and confluent presentations, both in the uniform (where the presentation is part of the input) and nonuniform setting? From Theorem 3 one obtains a LOGDCFL-upper bound. Equivalent questions were investigated in [2] in the context of McNaughton languages.

- Does there exist an automatic group or at least an automatic cancellative monoid with a P-complete word problem?

Concerning the undecidability result in Theorem 6 one might try to characterize more general classes of monoid properties that are in general undecidable for automatic monoids. Note that the property $\exists x \in \mathcal{M}: u x=v$ in $\mathcal{M}$ in Theorem 6 is an existential first-order property of the monoid $\mathcal{M}$. Thus, Theorem 6 can be rephrased by saying that there is a fixed automatic monoid with an undecidable existential first-order theory. Whether there exists a fixed automatic group with an undecidable existential first-order theory is again open. It is even open, whether the solvability of a single word equation over an automatic group is decidable. Let us mention here a result of [38]: Solvability of word equations over a torsion-free hyperbolic group is decidable.

\section{References}

1. G. Bauer and F. Otto. Finite complete rewriting systems and the complexity of the word problem. Acta Informatica, 21:521-540, 1984.

2. M. Beaudry, M. Holzer, G. Niemann, and F. Otto. McNaughton families of languages. Theoretical Computer Science, 290(3):1581-1628, 2003.

3. A. Blumensath and E. Grädel. Automatic structures. In Proceedings of the 15th Annual IEEE Symposium on Logic in Computer Science (LICS'2000), pages 51-62. IEEE Computer Society Press, 2000.

4. A. Blumensath and E. Grädel. Finite presentations of infinite structures: Automata and interpretations. Theory of Computing Systems, 37(6):641-674, 2004.

5. R. V. Book. Confluent and other types of Thue systems. Journal of the Association for Computing Machinery, 29(1):171-182, 1982.

6. R. V. Book, M. Jantzen, B. Monien, C. P. Ó'Dúnlaing, and C. Wrathall. On the complexity of word problems in certain Thue systems. In J. Gruska and M. Chytil, 
editors, Proceedings of the 10th International Symposium on Mathematical Foundations of Computer Science (MFCS'81), Štrbské Pleso (Czechoslovakia), number 118 in Lecture Notes in Computer Science, pages 216-223. Springer, 1981.

7. R. V. Book and F. Otto. String-Rewriting Systems. Springer, 1993.

8. J.-y. Cai. Parallel computation over hyperbolic groups. In Proceedings of the 24th Annual Symposium on Theory of Computing (STOC 92), pages 106-115. ACM Press, 1992.

9. H. Calbrix and T. Knapik. A string-rewriting characterization of Muller and Schupp's context-free graphs. In V. Arvind and R. Ramanujam, editors, Proceedings of the 18th International Conference on Foundations of Software Technology and Theoretical Computer Science, number 1530 in Lecture Notes in Computer Science, pages 331-342. Springer, 1999.

10. C. M. Campbell, E. F. Robertson, N. Ruškuc, and R. M. Thomas. Automatic semigroups. Theoretical Computer Science, 250(1-2):365-391, 2001.

11. K. J. Compton and C. W. Henson. A uniform method for proving lower bounds on the computational complexity of logical theories. Annals of Pure and Applied Logic, 48:1-79, 1990.

12. M. Coornaert, T. Delzant, and A. Papadopoulos. Géométrie et théorie des groupes. Number 1441 in Lecture Notes in Mathematics. Springer, 1990.

13. E. Dahlhaus and M. K. Warmuth. Membership for growing context-sensitive grammars is polynomial. Journal of Computer and System Sciences, 33:456-472, 1986.

14. A. Duncan and R. H. Gilman. Word hyperbolic semigroups. Mathematical Proceedings of the Cambridge Philosophical Society, 136:513-524, 2004.

15. D. B. A. Epstein, J. W. Cannon, D. F. Holt, S. V. F. Levy, M. S. Paterson, and W. P. Thurston. Word processing in groups. Jones and Bartlett, Boston, 1992.

16. M. Gromov. Hyperbolic groups. In S. M. Gersten, editor, Essays in Group Theory, number 8 in MSRI Publ., pages 75-263. Springer, 1987.

17. W. Hodges. Model Theory. Cambridge University Press, 1993.

18. M. Hoffmann. Automatic semigroups. PhD thesis, University of Leicester, Department of Mathematics and Computer Science, 2000.

19. M. Hoffmann, D. Kuske, F. Otto, and R. M. Thomas. Some relatives of automatic and hyperbolic groups. In Proceedings of the Thematic Term on Semigroups, Algorithms, Automata and Languages, pages 379-406. World Scientific, 2002.

20. M. Hoffmann and R. M. Thomas. Notions of automaticity in semigroups. Semigroup Forum, 66:337-367, 2003

21. D. Holt. Word-hyperbolic groups have real-time word problem. International Journal of Algebra and Computation, 10:221-227, 2000.

22. J. F. P. Hudson. Regular rewrite systems and automatic structures. In Semigroups, Automata and Languages, pages 145-152. World Scientific, 1998.

23. A. V. Kelarev and S. J. Quinn. A combinatorial property and Cayley graphs of semigroups. Semigroup Forum, 66(1):89-96, 2003.

24. B. Khoussainov and A. Nerode. Automatic presentations of structures. In $L C C$ : International Workshop on Logic and Computational Complexity, number 960 in Lecture Notes in Computer Science, pages 367-392, 1994.

25. T. Knapik and H. Calbrix. Thue specifications and their monadic second-order properties. Fundamenta Informaticae, 39:305-325, 1999.

26. D. Kuske and M. Lohrey. Logical aspects of Cayley-graphs: the group case. Annals 
of Pure and Applied Logic, 131(1-3):263-286, 2005.

27. D. Kuske and M. Lohrey. Logical aspects of Cayley-graphs: the monoid case. International Journal of Algebra and Computation, 2005. to appear.

28. M. Lohrey. Word problems and confluence problems for restricted semi-Thue systems. In L. Bachmair, editor, Proceedings of the 11th International Conference on Rewrite Techniques and Applications (RTA 2000), Norwich (UK), number 1833 in Lecture Notes in Computer Science, pages 172-186. Springer, 2000.

29. M. Lohrey. Word problems for 2-homogeneous monoids and symmetric logspace. In J. Sgall, A. Pultr, and P. Kolman, editors, Proceedings of the 26th International Symposium on Mathematical Foundations of Computer Science (MFCS 2001), Marianske Lazne (Czech Republic), number 2136 in Lecture Notes in Computer Science, pages 500-511. Springer, 2001.

30. M. Lohrey. Automatic structures of bounded degree. In Proceedings of the 10th International Conference on Logic for Programming, Artificial Intelligence, and Reasoning (LPAR 2003), Almaty (Kazakhstan), number 2850 in Lecture Notes in Artificial Intelligence, pages 344-358, 2003.

31. M. Lohrey. Decidability and complexity in automatic monoids. In C. S. Calude, E. Calude, and M. J. Dinneen, editors, Proceedings of the 8th International Conference on Developments in Language Theory (DLT 2004), Auckland (New Zealand), number 3340 in Lecture Notes in Computer Science, pages 308-320, 2004.

32. R. C. Lyndon and P. E. Schupp. Combinatorial Group Theory. Springer, 1977.

33. A. Markov. On the impossibility of certain algorithms in the theory of associative systems. Doklady Akademii Nauk SSSR, 55, 58:587-590, 353-356, 1947.

34. D. E. Muller and P. E. Schupp. Groups, the theory of ends, and context-free languages. Journal of Computer and System Sciences, 26:295-310, 1983.

35. D. E. Muller and P. E. Schupp. The theory of ends, pushdown automata, and second-order logic. Theoretical Computer Science, 37(1):51-75, 1985.

36. F. Otto and N. Ruškuc. Confluent monadic string-rewriting systems and automatic structures. Journal of Automata, Languages and Combinatorics, 6(3):375-388, 2001.

37. E. Post. Recursive unsolvability of a problem of Thue. Journal of Symbolic Logic, 12(1):1-11, 1947.

38. E. Rips and Z. Sela. Canonical representatives and equations in hyperbolic groups. Inventiones Mathematicae, 120:489-512, 1995.

39. W. L. Ruzzo. Tree-size bounded alternation. Journal of Computer and System Sciences, 21:218-235, 1980.

40. P. E. Schupp. Groups and graphs: Groups acting on trees, ends, and cancellation diagrams. Mathematical Intelligencer, 1:205-222, 1979.

41. G. Sénizergues. Formal languages and word-rewriting. In H. Comon and J.-P. Jouannaud, editors, Term Rewriting, French Spring School of Theoretical Computer Science, Font Romeux (France), number 909 in Lecture Notes in Computer Science, pages 75-94. Springer, 1993.

42. P. V. Silva and B. Steinberg. Extensions and submonoids of automatic monoids. Theoretical Computer Science, 289:727-754, 2002.

43. P. V. Silva and B. Steinberg. A geometric characterization of automatic monoids. The Quarterly Journal of Mathematics, 55:333-356, 2004.

44. I. H. Sudborough. On the tape complexity of deterministic context-free languages. Journal of the Association for Computing Machinery, 25(3):405-414, 1978. 
45. H. Venkateswaran. Properties that characterize LOGCFL. Journal of Computer and System Sciences, 43:380-404, 1991.

46. B. Zelinka. Graphs of semigroups. Casopis. Pest. Mat., 27:407-408, 1981. 